\title{
THERMAL MANIKIN AND ITS STABILITY FOR ACCURATE AND REPEATABLE MEASUREMENTS
}

\author{
Vujica Herzog, N. ${ }^{*}$; Zavec Pavlinic, D. ${ }^{* * *}$; Kuzmanovic, B. ${ }^{* * *} \&$ Buchmeister, B. \\ ${ }^{*}$ University of Maribor, Faculty of Mechanical Engineering, Smetanova 17, 2000 Maribor, Slovenia \\ ${ }^{* *}$ Titera, d.o.o., Obrtna 40, 9000 Murska Sobota, Slovenia \\ ${ }^{* * *}$ University of Novi Sad, Faculty of Technical Sciences, Trg Dositeja Obradovica 6, \\ 21000 Novi Sad, Serbia
}

E-Mail: natasa.vujica@um.si; daniela.zavec@um.si; kbogdan@uns.ac.rs; borut.buchmeister@um.si

\begin{abstract}
Thermal manikins are used for testing the thermal insulation of different types of protective clothing. Data about thermal insulation is required when new protective clothes are designed or for the optimization of the existing ones. Thermal insulation usually plays an important role when researching or developing optimal protective clothing used in hot environmental conditions. The aim is to develop protective clothing that will ensure the lowest possible thermal load for the user. To get accurate information about thermal insulation, the measuring system for its determination should be stable. One of the possibilities is to use a thermal manikin presenting the anatomic shape of the human body. The measurement accuracy and stability of the measuring system based on the thermal manikin are investigated and assessed on the basis of statistical analysis. Accurate measurements can be ensured with statistics. Only accurate data has an application value for the industrial development.

(Received in February 2016, accepted in June 2016. This paper was with the authors 1 month for 1 revision.)
\end{abstract}

Key Words: Thermal Insulation, Thermal Manikin, Measurement Validity and Reliability, System Stability Assessment

\section{INTRODUCTION}

Safety is not only the first component of values statement, but is always the first consideration in system design requirements, work practices and company policy. Knowledge, experience and knowhow are shared resources between the different applications to bring the best available solutions, when a high risk of suffering critical interaction with hazardous environment is present. To ensure reliable and safe systems different products or methods are available, for example: protection systems [1-3], safety sensors, controllers, scanners [4], correct design of devices (ergonomics) [5, 6], application of innovative principles [7], heat transfer simulation for cosmetic products [8], virtual human models [9] etc.

Thermal insulation is one of the most important parameters that should be considered when developing protective clothing intended for use in an extreme environment with high temperatures. It describes the amount of heat transfer through the layers of textile materials of which the particular protective clothing is made.

To determine thermal insulation a thermal manikin can be used, which best illustrates the anatomic shape of the human body. By using this measurement system, thermal insulation can be defined for an entire garment, for a clothing system or for a certain segment only ('regional'). Regional thermal insulation is important when heat transfer through the layers of textile material only needs to be ensured in specific parts or segments of the garment. The thermal manikin that we used has 19 segments [2, 10-12]. By measuring the temperature and heat flow in the individual segments it is possible to define regional thermal insulation.

Thermal manikins have a long tradition. Representing the anatomic shape of the human body, they were first used in the early 1940s [11] when a simple one-segment manikin made from copper was used for the needs of the American army. The idea of development and 
manufacture was based on testing firearms and protective equipment in World War II. After that, several more sophisticated manikins were developed [13, 14]. Nowadays there are more than 100 thermal manikins in use worldwide [15-18]. Each new example represents a significant improvement in technique and approach [19-23].

\section{RESEARCH PROBLEM}

The most important result that we are looking for when carrying out tests using a thermal manikin is thermal insulation, which increases with each added textile layer inside the clothing system (Fig. 1). In order to convert the measurements into useful data, the thermal resistance $\left(R_{t i}\right)$ and total clothing thermal insulation value $\left(I_{i}\right)$ can be calculated automatically for each of the nineteen segments separately or for all the segments together.

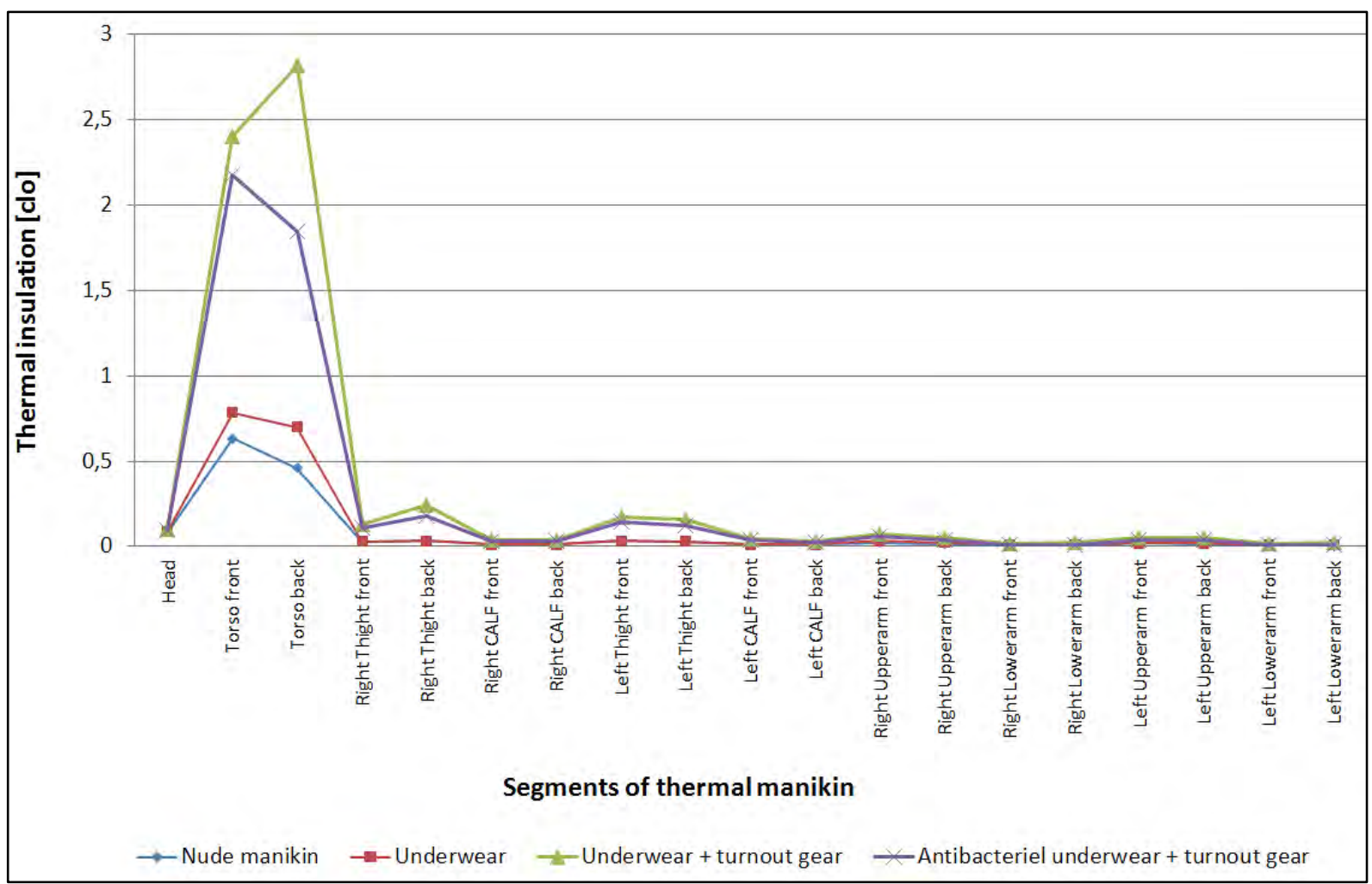

Figure 1: Thermal insulation.

Thermal resistance of a particular manikin segment in clo is calculated as follows:

$$
R_{t i}=\frac{T_{s, i}-T_{a m b}}{0.155 Q_{i}}
$$

where:

$T_{S, i} \quad$ - surface temperature of a segment I (K)

$T_{a m b} \quad$ - ambient temperature (K)

$Q_{i} \quad$ - rate of heat transfer $\left(\mathrm{Wm}^{-2}\right)$

0.155 - factor for conversion from $\left(\mathrm{m}^{2} \mathrm{~K} \mathrm{~W}^{-1}\right)$ to (clo)

The total clothing thermal insulation of a manikin segment in clo is calculated as follows:

where:

$$
I_{i}=\frac{A_{i}\left(T_{s, i}-T_{a m b}\right)}{0.155 Q_{i}}
$$

$A_{i} \quad-$ surface of the particular manikin segment $\left(\mathrm{m}^{2}\right)$. 
Measurements using a thermal manikin are very suitable for determining the thermal insulation of protective and working clothes. Since textile materials are not homogeneous, the same measuring conditions (ambient temperature and humidity) should be ensured every time when measurements are taken. It is also very important that the measuring system - in our case a thermal manikin - is stable.

For data capturing a suitable user interface was developed. The user interface is designed to ensure a good overview of all the crucial data, temperature and power. It enables simple and controlled testing according to the ISO 15831 (2004) [23] standard. Each of the nineteen segments communicates with the control unit and the data on the user interface is shown in real time (Fig. 2). If a failure occurs, the operator can see which segment is not functioning.

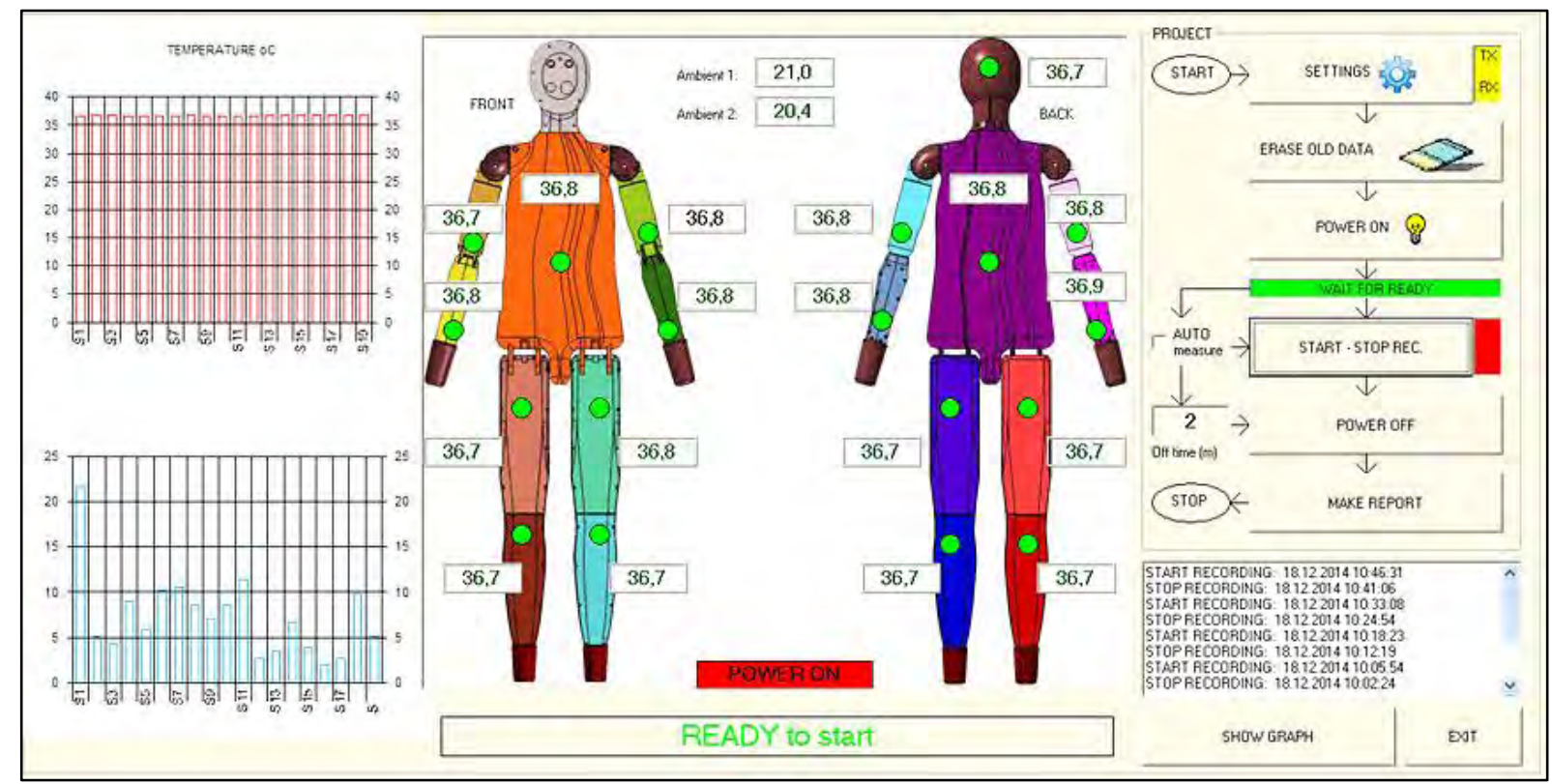

Figure 2: Control panel of the TmpTelo interface.

The control panel presents all nineteen segments with measured regional temperatures. On the left side there is also a graphic presentation of reached temperature and current power (in $\%$ of the maximum possible load) conducted to a certain segment. The right-hand side allows measurements according to the ISO 15831 (2004) standard, with a documented report.

With this user interface, the measured results of each of the nineteen segments can be displayed in real time (Fig. 3). Any impact from the environment can be observed both while increasing the surface temperature and during measurement. All the measured data can be saved or printed out.

The heating of all the nineteen segments from ambient room temperature to a set temperature of $36^{\circ} \mathrm{C}$ is presented. The tags from 1 to 19 present the manikin segments and tag 20 presents temperature of the environment. Due to the different masses and applied power, the segments are not heated at the same rates during the pre-heating phase. The measurement began in stable conditions.

The following four sets of thermal conditions with different clothes were tested using a thermal manikin (Fig. 4):

1. Naked manikin

2. Thermal manikin with underwear

3. Firefighter clothing with flame retardant uniform

4. Firefighter clothing with antibacterial protection 


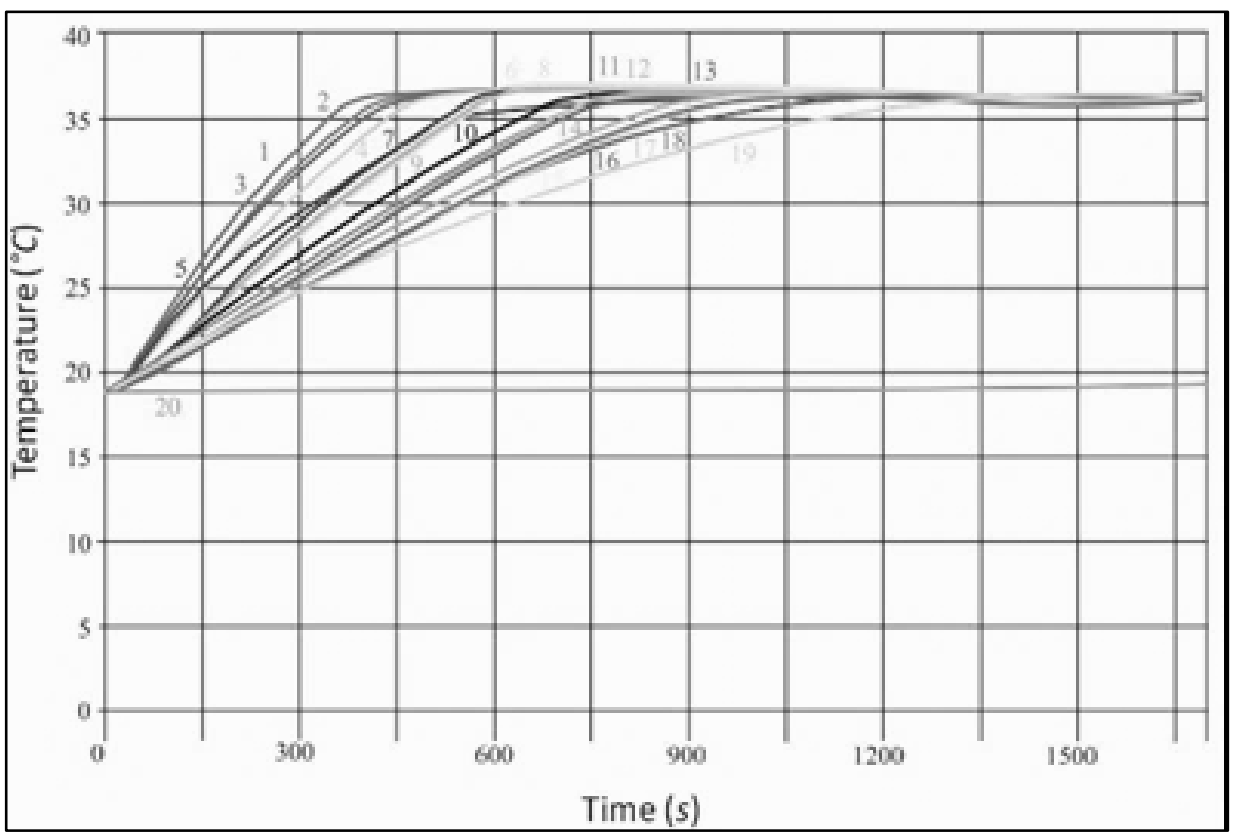

Figure 3: Graphical presentation of real time measurements.
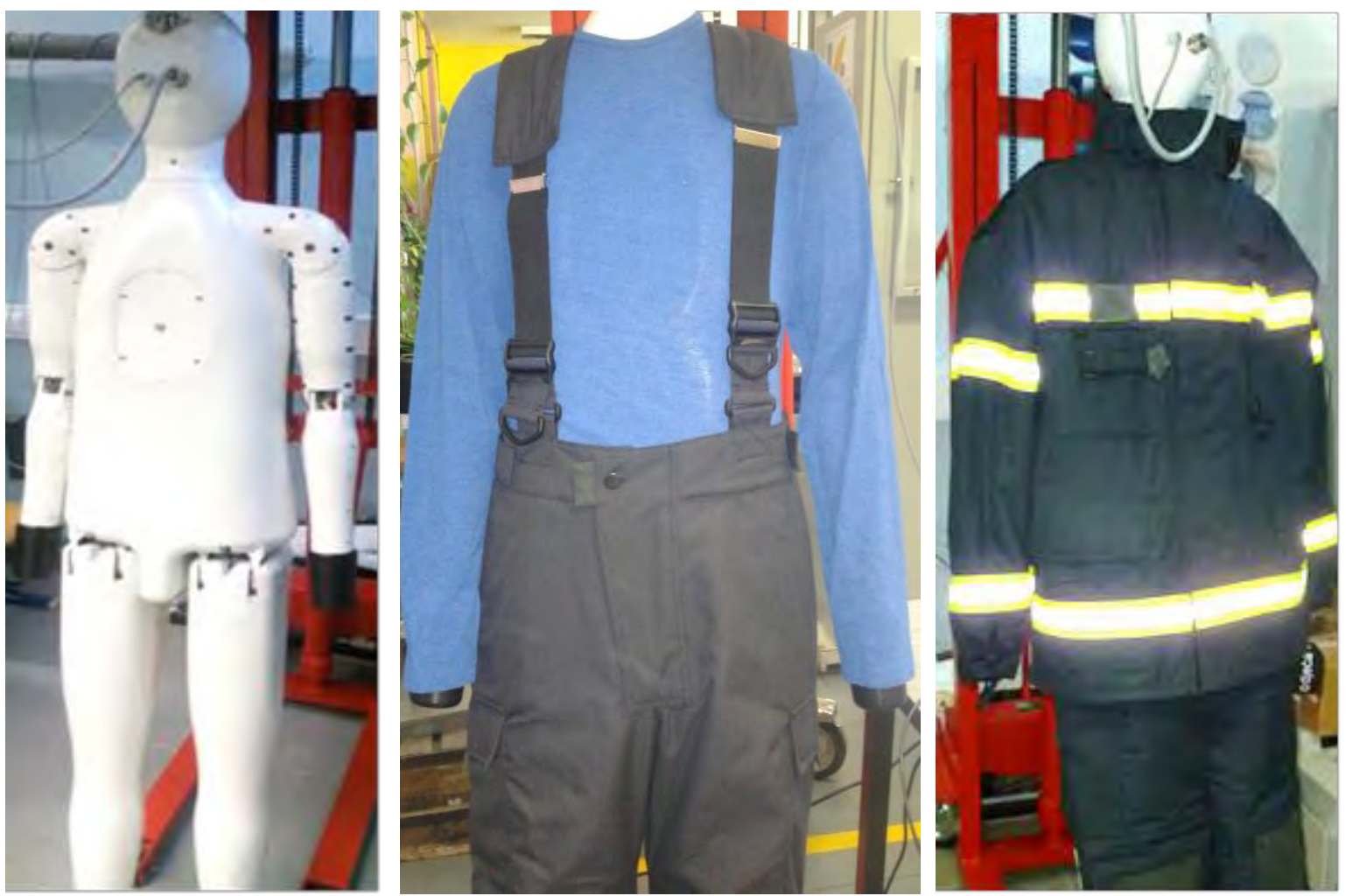

Figure 4: Thermal manikin - naked and dressed (with underwear and in a firefighter suit).

For the analysis of the problem, descriptive statistics and Paired sample $t$-test as multivariate analysis of variance were used.

\section{RESEARCH FRAMEWORK}

Using a thermal manikin, the temperature and heat flux were measured. Based on the known areas $\left(\mathrm{cm}^{2}\right)$ of individual segments, the thermal resistance was calculated, Eq. (1). Then the 
thermal insulation was calculated, Eq. (2) in units called clo $\left(1 \mathrm{clo}=0,155 \mathrm{~m}^{2 \circ} \mathrm{C} / \mathrm{W}\right)$. Since the values of thermal insulation and temperature are in linear combination, the relationships between three variables were observed: temperature in linear combination with thermal insulation, heat flow and thermal power (Fig. 1). Fig. 1 presents our overall research framework concerning the system reliability and validity for thermal insulation through two hypotheses stated in the null form. Since the heat flow and the thermal power are in complete linear combination (correlation $=1$ ), only two hypotheses stated in the null form were tested:

Hypothesis 1: There is a strong and positive relationship between temperature and heat flow.

Hypothesis 2: There is a strong and positive relationship between temperature and power.

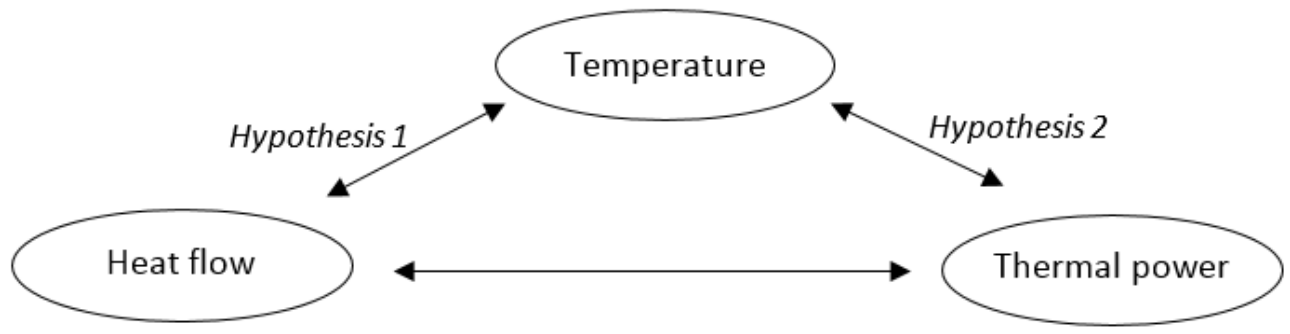

Figure 5: Conceptual framework.

\section{METHODOLOGY}

The presented research was divided into the following phases:

- a wide-ranging data collection was performed on the 19 observed segments of the thermal manikin, measuring three values: temperature, heat flow and power. Seventy-five measurements were performed on a naked manikin, 122 on a thermal manikin with underwear, 132 on a manikin dressed in firefighter clothes with a flame retardant and 150 measures on a manikin dressed in firefighter clothes with antibacterial protection;

- the research hypotheses were tested using SPSS statistical program package;

- the resulting data was subjected to reliability and validity analyses to determine the measurement properties, and then analysed using uni- and multivariate statistical techniques.

Since the aim of quantitative research is to get familiarized with the concept to be studied and to generate the hypothesis to be tested, the emphasis of research was put on:

a. the facts and causes of behaviour [24],

b. the information in the form of numbers that can be quantified and summarized,

c. the mathematical process used for analysing numerical data,

d. the final result expressed in statistical terminology [25].

\subsection{Measurement error}

The use of multiple variables and the reliance on their combination in multivariate techniques also draws attention to measurement error [26]. Measurement error is the degree to which the observed values are not representatives of the 'true' values. Measurement errors have many causes, ranging from data entry errors to the imprecision of the measurement and also the inability of respondents to provide information accurately. For this reason, all variables used in multivariate techniques can have some degree of measurement error. To assess the degree of error present in any measurement, the validity and reliability of the measure should be addressed. 


\subsection{Validity}

The validity of a measure refers to the extent to which it measures what is intended to be measured [27]. In other words, validity is the degree to which a measure accurately represents what it is supposed to do [26]. Ensuring validity starts with thorough understanding of what is to be measured and then making the measurement as 'correct' and accurate as possible. If validity is ensured, the researcher must still consider the reliability of the measurement. Validity does not guarantee reliability, and vice versa. A measure may be accurate (valid) but not consistent (reliable). Also, it may be quite consistent but not accurate. Thus, validity and reliability are two separate though interrelated conditions.

\subsection{Reliability}

Reliability is the degree to which the observed variable measures the 'true' value and is 'error free'; it is, therefore, the opposite of measurement error [26]. In more formal terms, reliability is the extent to which a set of two or more indicators 'share' their measurement of a construct. The indicators of highly reliable constructs are highly intercorrelated, showing that they are all measuring the same latent construct. With decreased reliability, the indicators become less consistent and thus poorer indicators of the latent construct. Reliability can be computed as 1.0 minus the measurement error.

Kirk and Miller [28] identify three types of reliability in quantitative research, which relate to:

- the degree to which a measurement, given repeatedly, remains the same,

- the stability of a measurement over time and

- the similarity of measurements within a given time period.

\subsection{Paired sample $t$-test technique}

The Paired sample $t$-test is a technique that belongs to a group of multivariate analysis of variance [26]. Multivariate analysis of variance is the multivariate extension of the univariate techniques for assessing the differences between group means. The $t$-test assesses the statistical significance of the difference between two independent sample means. To determine whether the two messages are viewed differently (meaning that the treatment has an effect) a $t$ statistic is calculated. The $t$ statistic is the ratio of the difference between the sample means $\left(\mu_{1}-\mu_{2}\right)$ and their standard error. The standard error is an estimate of the difference between means to be expected because of a sampling error, rather than real differences between means.

\section{ANALYSIS AND RESULTS}

\subsection{Descriptive statistics}

In the following Tables I to IV, researched variables for all four types of thermal conditions with different clothing (naked manikin, thermal manikin with underwear, firefighter clothing with a flame retardant and firefighter clothing with antibacterial protection) are explained by:

- the minimum and maximum values,

- the mean value of each variable,

- the standard error and standard deviation.

As we can see in Tables I and II, there are some deviations within data on specific measuring points (marked in bold). 
Table I: Naked manikin data.

\begin{tabular}{|c|c|c|c|c|c|c|c|c|c|c|c|c|c|c|c|c|c|}
\hline & Min & Max & Mean & St.Err & St.D. & & Min & Max & Mean & St.Err & St.D. & & Min & Max & Mean & St.Err & St.D. \\
\hline $\mathrm{t} 1$ & 36,8 & 37,1 & 36,96 & 009 & 081 & pw1 & 22,22 & 26,82 & 24,33 &, 143 & 1,24 & pwcml & 154,4 & 186,3 & 169,06 & ,998 & 8,65 \\
\hline t2 & 36,8 & 37,0 & 36,90 & 007 &, 060 & pw 2 & 15,38 & 21,76 & 18,83 &, 182 & 1,57 & pwcm2 & 44,1 & 62,4 & 53,99 &, 521 & 4,51 \\
\hline $\mathrm{t} 3$ & 36,9 & 37,0 & 36,98 &, 004 & 039 & pw3 & 18,32 & 29,40 & 22,14 & 318 & 2,75 & pwcm3 & 56,2 & 90,2 & 67,93 & ,974 & 8,44 \\
\hline $\mathrm{t} 4$ & 36,8 & 37,1 & 37,01 &, 010 & 089 & pw4 & 17,36 & 34,90 & 23,38 & 626 & 5,42 & pwcm4 & 204,2 & 410,6 & 275,04 & 7,368 & 63,80 \\
\hline t5 & 36,8 & 37,2 & 37,02 &, 009 & 079 & pw5 & 13,14 & 31,80 & 19,27 & 623 & 5,40 & pwcm5 & 154,6 & 374,1 & 226,77 & 7,337 & 63,54 \\
\hline t6 & 36,9 & 37,1 & 37,00 & 010 & 088 & pw6 & 14,90 & 29,40 & 19,51 & ,457 & 3,96 & pwcm6 & 289,4 & 571,1 & 379,05 & 8,885 & 76,95 \\
\hline $\mathrm{t} 7$ & 36,9 & 37,1 & 37,02 &, 007 &, 062 & pw7 & 16,58 & 27,50 & 20,29 &, 378 & 3,27 & pwcm7 & 322,1 & 534,2 & 394,17 & 7,349 & 63,64 \\
\hline t8 & 36,9 & 37,1 & 37,04 &, 007 &, 064 & pw8 & 14,50 & 31,38 & 20,24 &, 558 & 4,84 & pwcm8 & 170,6 & 369,2 & 238,20 & 6,572 & 56,91 \\
\hline t9 & 36,8 & 37,1 & 37,03 &, 008 &, 074 & pw9 & 17,30 & 32,06 & 21,85 &, 485 & 4,20 & pwcm9 & 203,5 & 377,2 & 257,09 & 5,713 & 49,47 \\
\hline $\mathrm{t} 10$ & 36,9 & 37,1 & 37,01 &, 010 & 087 & pw10 & 14,02 & 26,12 & 17,33 &, 343 & 2,97 & pwcm 10 & 272,3 & 507,4 & 336,75 & 6,669 & 57,76 \\
\hline $\mathrm{t} 11$ & 36,9 & 37,1 & 37,02 &, 008 & ,069 & pw11 & 18,64 & 30,84 & 22,84 & ,336 & 2,91 & pwcml1 & 362,1 & 599,1 & 443,78 & 6,536 & 56,61 \\
\hline $\mathrm{t} 12$ & 36,8 & 37,0 & 36,86 &, 007 &, 063 & pw12 & 5,72 & 11,00 & 7,96 &, 149 & 1,29 & pwcm 12 & 139,0 & 267,4 & 193,45 & 3,636 & 31,48 \\
\hline $\mathrm{t} 13$ & 36,8 & 37,0 & 36,88 &, 007 &, 065 & pw13 & 9,80 & 14,52 & 12,38 &, 141 & 1,22 & pwo & 238,2 & 352,9 & 301,15 & 3,429 & 29,70 \\
\hline $\mathrm{t} 14$ & 36,8 & 37,2 & 36,97 &, 015 &, 132 & pw14 & 13,46 & 16,90 & 15,33 &, 127 & 1,09 & pwc & 376,7 & 472,9 & 429,17 & 3,554 & 30,78 \\
\hline $\mathrm{t} 15$ & 36,8 & 37,2 & 36,99 &, 015 &, 128 & pw15 & 12,42 & 16,10 & 14,29 &, 109 & ,94 & pwo & 400,6 & 519,3 & 461,19 & 3,513 & 30,42 \\
\hline $\mathrm{t} 16$ & 36,8 & 37,2 & 36,96 &, 015 &, 134 & pw16 & 7,64 & 11,94 & 10,29 &, 121 & 1,05 & pwcm 16 & 185,7 & 290,2 & 250,20 & 2,952 & 25,57 \\
\hline $\mathrm{t} 17$ & 36,8 & 37,1 & 36,96 &, 011 & 099 & pw17 & 10,60 & 15,22 & 12,62 &, 129 & 1,12 & pwcm 17 & 257,6 & 369,9 & 306,88 & 3,153 & 27,30 \\
\hline $\mathrm{t} 18$ & 36,8 & 37,2 & 37,01 &, 017 &, 145 & pw18 & 13,22 & 18,40 & 15,79 &, 154 & 1,33 & pwcm 18 & 369,9 & 514,9 & 442,13 & 4,309 & 37,32 \\
\hline $\mathrm{t} 19$ & 36,8 & 37,3 & 37,08 &, 018 &, 154 & pw19 & 13,70 & 21,60 & 16,96 &, 180 & 1,56 & pwcm19 & 441,9 & 696,8 & 547,21 & 5,809 & 50,31 \\
\hline
\end{tabular}

Table II: Manikin with underwear data.

\begin{tabular}{|c|c|c|c|c|c|c|c|c|c|c|c|c|c|c|c|c|c|}
\hline & Min & Max & Mean & St.Err & St.D. & & Min & Max & Mean & StErr & St.D. & & Min & Max & Mean & St.Err & St.D. \\
\hline tl & 36,8 & 36,8 & 36,80 & 001 & 015 & pw1 & 21,4 & 24,2 & 23,81 & 041 &, 453 & pwcml & 148,8 & 168,2 & 165,43 & ,285 & 3,148 \\
\hline $\mathrm{t} 2$ & 36,7 & 36,9 & 36,83 & ,005 & 055 & pw 2 & 11,0 & 18,8 & 14,37 & 202 & 2,239 & pwem2 & 31,5 & 53,8 & 41,20 &, 581 & 6,418 \\
\hline t3 & 36,7 & 36,9 & 36,84 & ,005 & 065 & pw3 & 9,6 & 21,7 & 14,08 & ,303 & 3,356 & pwcm3 & 29,5 & 66,7 & 43,21 & ,932 & 10,295 \\
\hline $\mathrm{t} 4$ & 36,5 & 36,9 & 36,73 &, 008 & ,092 & pw4 & 18,0 & 29,2 & 23,04 & 231 & 2,554 & pwem4 & 211,7 & 343,2 & 271,03 & 2,720 & 30,052 \\
\hline t5 & 36,5 & 36,9 & 36,74 & 010 & 117 & pw5 & 15,7 & 27,5 & 20,66 & 279 & 3,092 & pwcm5 & 184,7 & 323,5 & 243,07 & 3,293 & 36,374 \\
\hline t6 & 36,4 & 36,9 & 36,72 &, 012 & 142 & pw6 & 18,4 & 31,1 & 23,44 &, 330 & 3,646 & pwem6 & 357,4 & 603,7 & 455,40 & 6,412 & 70,833 \\
\hline t7 & 36,5 & 36,9 & 36,76 &, 011 &, 125 & pw7 & 17,3 & 30,6 & 22,76 &, 332 & 3,673 & pwcm7 & 336,4 & 594,4 & 442,11 & 6,460 & 71,356 \\
\hline t8 & 36,5 & 36,9 & 36,73 & ,009 & 102 & pw8 & 16,1 & 27,4 & 20,63 &, 256 & 2,834 & pwcm8 & 189,4 & 322,1 & 242,79 & 3,018 & 33,340 \\
\hline t9 & 36,6 & 36,9 & 36,77 & 009 & 104 & pw9 & 16,9 & 27,1 & 22,17 & ,288 & 3,189 & pwcm9 & 198,8 & 318,8 & 260,86 & 3,396 & 37,518 \\
\hline $\mathrm{t} 10$ & 36,4 & 36,9 & 36,71 &, 010 &, 115 & pw10 & 15,9 & 27,5 & 21,15 & ,264 & 2,926 & pwem 10 & 309,6 & 534,5 & 410,89 & 5,146 & 56,848 \\
\hline t11 & 36,5 & 36,9 & 36,74 &, 010 &, 116 & pw11 & 20,8 & 32,5 & 25,69 & 289 & 3,198 & pwcml1 & 404,0 & 631,3 & 499,07 & 5,624 & 62,123 \\
\hline t12 & 36,8 & 36,9 & 36,84 & ,004 & 046 & pw12 & 1,7 & 8,2 & 4,69 & 140 & 1,546 & pwcm 12 & 42,8 & 199,3 & 114,09 & 3,403 & 37,593 \\
\hline $\mathrm{t} 13$ & 36,8 & 37,0 & 36,85 & ,004 & 049 & pw13 & 4,5 & 12,9 & 7,36 &, 130 & 1,441 & pwc & 110,3 & 313,5 & 178,92 & 3,170 & 35,018 \\
\hline $\mathrm{t} 14$ & 36,7 & 36,9 & 36,82 &, 005 &, 060 & pw14 & 9,4 & 17,8 & 13,27 & ,204 & 2,262 & pwo & 263,0 & 498,7 & 371,60 & 5,732 & 63,314 \\
\hline $\mathrm{t} 15$ & 36,6 & 36,9 & 36,82 & ,008 & 088 & pw15 & 4,3 & 15,3 & 9,06 & ,235 & 2,602 & & 138,7 & 493,5 & 292,22 & 7,599 & 83,940 \\
\hline t16 & 36,8 & 36,9 & 36,84 &, 004 &, 043 & pw16 & 5,3 & 11,4 & 8,05 &, 151 & 1,668 & pwcm 16 & 129,8 & 277,1 & 195,62 & 3,670 & 40,546 \\
\hline t17 & 36,8 & 36,9 & 36,85 &, 004 &, 044 & pw17 & 5,5 & 12,9 & 8,80 &, 173 & 1,919 & pwcm 17 & 135,6 & 313,5 & 214,00 & 4,225 & 46,668 \\
\hline $\mathrm{t} 18$ & 36,8 & 36,9 & 36,84 &, 004 &, 045 & pw18 & 11,0 & 17,6 & 13,74 &, 174 & 1,927 & pwcm 18 & 307,8 & 492,5 & 384,72 & 4,883 & 53,938 \\
\hline t19 & 36,7 & 36,9 & 36,84 & ,006 & 063 & pw19 & 8,2 & 18,1 & 12,06 & 198 & 2,194 & pwcm19 & 267,0 & 585,8 & 389,12 & 6,407 & 70,769 \\
\hline
\end{tabular}

\subsection{Paired sample $t$-test}

The basic idea of the Paired sample $t$-test is simple. If the treatment had no effect, the average difference between the measurements is equal to 0 and the null hypothesis holds. On the other hand, if the treatment did have an effect (intended or unintended), the average difference is not 0 and the null hypothesis is rejected.

The first analysis (Table V) performed on the naked manikin shows differences between the observed measurement points. Pairs 3, 6, 7, 9 and 15 show little correlation between temperature and heat flow, so for this first measurement set the null hypothesis cannot be accepted for all the measurement points.

A more specific view shows that for 12 measurement points, the null hypothesis can be accepted with $99 \%$ probability but, as mentioned before, for other measurement points the hypothesis cannot be accepted. 
Vujica Herzog, Zavec Pavlinic, Kuzmanovic, Buchmeister: Thermal Manikin and Its ...

Table III: Firefighter clothing with a flame retardant data.

\begin{tabular}{|c|c|c|c|c|c|c|c|c|c|c|c|c|c|c|c|c|c|}
\hline & Min & Max & Mean & St.Err & St.D. & & Min & $\operatorname{Max}$ & Mean & St.Err & St.D. & & Min & Max & Mean & StErr & St.D. \\
\hline t1 & 36,8 & 36,9 & 36,84 & ,004 & ,042 & pw1 & 18,2 & 23,9 & 21,29 & ,134 & 1,550 & pwcml & 126,7 & 166,0 & 147,96 & ,937 & 10,772 \\
\hline t2 & 36,8 & 36,9 & 36,86 &, 003 & 038 & pw 2 & 2,8 & 9,7 & 5,62 & , 159 & 1,827 & pwcm2 & 8,0 & 27,8 & 16,13 & ,456 & 5,239 \\
\hline t3 & 36,8 & 37,0 & 36,87 & ,005 & 059 & pw3 & 1,6 & 12,2 & 4,57 & 196 & 2,258 & pwcm3 & 5,1 & 37,4 & 14,02 & 603 & 6,928 \\
\hline t4 & 36,8 & 37,1 & 36,90 & ,009 & , 108 & pw4 & 2,4 & 10,6 & 6,04 &, 117 & 1,353 & pwcm4 & 28,2 & 124,7 & 71,11 & 1,386 & 15,925 \\
\hline t5 & 36,8 & 37,1 & 36,89 & ,009 &, 111 & pw5 & 0,2 & 5,5 & 3,25 & ,096 & 1,107 & pwcm5 & 2,8 & 64,7 & 38,25 & 1,134 & 13,035 \\
\hline t6 & 36,7 & 37,0 & 36,86 & ,008 &, 101 & pw6 & 4,7 & 11,0 & 8,04 &, 119 & 1,371 & pwcm6 & 91,2 & 213,6 & 156,31 & 2,318 & 26,641 \\
\hline t7 & 36,8 & 37,0 & 36,87 & ,007 & 087 & pw7 & 5,0 & 10,2 & 7,99 & ,094 & 1,086 & pwcm7 & 97,5 & 198,1 & 155,20 & 1,836 & 21,096 \\
\hline t8 & 36,8 & 37,1 & 36,90 & ,009 &, 106 & pw8 & 1,2 & 8,6 & 4,63 &, 116 & 1,336 & pwcm8 & 14,1 & 101,1 & 54,56 & 1,368 & 15,725 \\
\hline t9 & 36,8 & 37,1 & 36,90 & ,009 & , 107 & pw9 & 1,6 & 9,5 & 5,07 & ,116 & 1,336 & pwcm9 & 18,8 & 112,4 & 59,70 & 1,368 & 15,719 \\
\hline $\mathrm{t} 10$ & 36,7 & 37,0 & 36,85 & ,008 & ,098 & pw10 & 2,7 & 8,8 & 6,22 &, 125 & 1,443 & pwcm 10 & 52,4 & 171,7 & 120,98 & 2,441 & 28,048 \\
\hline t11 & 36,8 & 37,1 & 36,89 & ,009 &, 103 & pw11 & 6,2 & 12,0 & 9,62 & 100 & 1,158 & pwcm11 & 120,4 & 234,2 & 186,98 & 1,958 & 22,496 \\
\hline $\mathrm{t} 12$ & 36,7 & 36,9 & 36,82 &, 004 &, 041 & pw12 & 0,0 & 7,1 & 2,78 &, 119 & 1,374 & pwcm12 & 0,0 & 172,5 & 67,69 & 2,908 & 33,411 \\
\hline $\mathrm{t} 13$ & 36,7 & 36,9 & 36,86 &, 004 &, 044 & pw13 & 1,6 & 10,2 & 4,01 &, 160 & 1,843 & pwcm13 & 40,8 & 247,9 & 97,50 & 3,899 & 44,805 \\
\hline $\mathrm{t} 14$ & 36,7 & 37,0 & 36,82 & ,006 & ,066 & pw14 & 4,3 & 14,9 & 8,47 & ,237 & 2,728 & pwcm 14 & 122,5 & 417,0 & 237,14 & 6,647 & 76,369 \\
\hline $\mathrm{t} 15$ & 36,5 & 36,9 & 36,81 & ,007 & 089 & pw15 & 1,6 & 17,3 & 5,78 & 311 & 3,582 & pwcm 15 & 51,6 & 558,0 & 186,65 & 10,058 & 115,568 \\
\hline $\mathrm{t} 16$ & 36,8 & 36,9 & 36,86 &, 003 & 038 & pw16 & 1,2 & 7,8 & 3,85 &, 138 & 1,593 & pwcm 16 & 31,1 & 189,5 & 93,58 & 3,371 & 38,732 \\
\hline $\mathrm{t} 17$ & 36,8 & 36,9 & 36,85 &, 003 & 042 & pw17 & 1,0 & 8,6 & 3,68 &, 141 & 1,620 & pwcm17 & 25,2 & 209,0 & 89,57 & 3,429 & 39,401 \\
\hline $\mathrm{t} 18$ & 36,7 & 37,0 & 36,84 &, 005 & 063 & pw18 & 4,9 & 16,5 & 8,46 & ,238 & 2,742 & pwcm 18 & 138,2 & 461,7 & 236,82 & 6,681 & 76,759 \\
\hline t19 & 36,7 & 36,9 & 36,83 &, 005 &, 061 & pw19 & 1,3 & 13,7 & 5,42 & ,276 & 3,172 & pwcm19 & 43,8 & 441,9 & 174,85 & 8,906 & 102,327 \\
\hline
\end{tabular}

Table IV: Firefighter clothing with antibacterial protection data.

\begin{tabular}{|c|c|c|c|c|c|c|c|c|c|c|c|c|c|c|c|c|c|}
\hline & Min & Max & Mean & St.Err & St.D. & & Min & $\operatorname{Max}$ & Mean & St.Err & St.D. & & Min & Max & Mean & St.Err & St.D. \\
\hline $\mathrm{t} 1$ & 36,7 & 36,8 & 36,78 &, 002 & 030 & pwl & 20,4 & 23,5 & 21,23 & ,069 & ,845 & pwcml & 142,2 & 163,2 & 147,52 & ,479 & 5,873 \\
\hline t2 & 36,7 & 36,8 & 36,77 & ,003 & 037 & pw 2 & 4,5 & 7,7 & 5,29 & ,087 & 1,070 & pwcm2 & 13,0 & 22,3 & 15,17 &, 250 & 3,069 \\
\hline t3 & 36,6 & 36,9 & 36,75 & ,005 & 070 & pw3 & 1,0 & 9,4 & 5,05 & ,159 & 1,957 & pwcm3 & 3,1 & 28,8 & 15,52 & ,490 & 6,004 \\
\hline t4 & 36,8 & 36,8 & 36,80 & ,001 & 016 & pw4 & 4,1 & 7,0 & 6,56 &, 040 & 499 & pwcm4 & 48,7 & 82,5 & 77,29 & ,479 & 5,872 \\
\hline t5 & 36,7 & 36,8 & 36,79 & ,001 & 014 & pw5 & 3,7 & 6,8 & 3,99 & ,034 & ,427 & pwcm5 & 44,0 & 80,7 & 46,95 &, 410 & 5,024 \\
\hline t6 & 36,7 & 36,8 & 36,77 &, 002 & 036 & pw6 & 7,1 & 10,5 & 8,15 & ,083 & 1,020 & pwcm6 & 139,4 & 204,3 & 158,33 & 1,618 & 19,825 \\
\hline t7 & 36,8 & 36,9 & 36,81 &, 002 & 031 & pw7 & 5,3 & 8,9 & 8,00 & ,079 & ,979 & pwcm7 & 103,7 & 173,2 & 155,48 & 1,553 & 19,023 \\
\hline t8 & 36,8 & 36,8 & 36,80 &, 00 &, 00 & pw8 & 5,1 & 5,1 & 5,10 &, 00 & ,00 & pwcm8 & 60,0 & 60,0 & 60,00 &, 00 & ,00 \\
\hline t9 & 36,7 & 36,8 & 36,78 &, 002 & 029 & pw9 & 5,1 & 8,3 & 5,85 &, 066 & ,814 & pwcm9 & 60,9 & 98,3 & 68,80 &, 782 & 9,578 \\
\hline $\mathrm{t} 10$ & 36,7 & 36,8 & 36,77 &, 003 &, 036 & pw10 & 5,1 & 8,5 & 6,25 &, 085 & 1,047 & pwcm 10 & 100,6 & 165,5 & 121,40 & 1,661 & 20,354 \\
\hline t11 & 36,8 & 36,8 & 36,80 &, 000 & 009 & pw11 & 8,2 & 10,2 & 10,14 &, 022 & ,277 & pwcm11 & 160,4 & 198,1 & 197,04 & ,440 & 5,393 \\
\hline $\mathrm{t} 12$ & 36,6 & 36,8 & 36,78 &, 004 & 051 & pw12 & 0,4 & 7,5 & 2,50 &, 110 & 1,351 & pwcm12 & 9,7 & 182,3 & 60,79 & 2,682 & 32,849 \\
\hline $\mathrm{t} 13$ & 36,6 & 36,9 & 36,77 & ,004 &, 054 & pw13 & 0,8 & 9,0 & 4,11 &, 124 & 1,520 & pwcm 13 & 19,4 & 218,7 & 99,89 & 3,017 & 36,954 \\
\hline $\mathrm{t} 14$ & 36,5 & 36,9 & 36,79 & ,007 & ,086 & pw14 & 4,3 & 18,4 & 8,68 &, 253 & 3,107 & pwcm14 & 122,5 & 514,9 & 242,88 & 7,100 & 86,964 \\
\hline $\mathrm{t} 15$ & 36,5 & 36,9 & 36,81 & ,007 &, 086 & pw15 & 2,0 & 15,9 & 6,32 & ,269 & 3,303 & pwcm 15 & 64,5 & 513,5 & 203,89 & 8,700 & 106,563 \\
\hline $\mathrm{t} 16$ & 36,6 & 36,9 & 36,80 &, 005 &, 071 & pw16 & 0,4 & 9,4 & 3,79 & ,168 & 2,063 & pwcm 16 & 11,6 & 228,4 & 92,15 & 4,094 & 50,149 \\
\hline $\mathrm{t} 17$ & 36,6 & 36,9 & 36,77 &, 005 &, 066 & pw17 & 0,2 & 8,6 & 3,85 &, 151 & 1,852 & pwcm 17 & 5,8 & 209,0 & 93,74 & 3,675 & 45,018 \\
\hline $\mathrm{t} 18$ & 36,5 & 36,9 & 36,79 &, 009 &, 117 & pw18 & 4,3 & 18,4 & 8,87 &, 311 & 3,820 & pwcm 18 & 122,5 & 514,9 & 248,28 & 8,730 & 106,922 \\
\hline $\mathrm{t} 19$ & 36,5 & 36,9 & 36,78 & 009 &, 120 & pw19 & 1,6 & 15,7 & 6,40 & ,334 & 4,101 & pwcm 19 & 54,2 & 506,4 & 206,58 & 10,803 & 132,314 \\
\hline
\end{tabular}

The analysis (Table VI) for the tests with the manikin with underwear gave better results, showing very high correlation between temperature and heat flow; consequently, the null hypothesis can be accepted with $99 \%$ probability, except for pair 19. 
Table V: Correlations between paired samples for the naked manikin.

\begin{tabular}{|ll|c|c|c|}
\cline { 2 - 4 } \multicolumn{1}{c|}{} & $\boldsymbol{N}$ & Correlation & Sig. \\
\hline Pair 1 & t1 \& pw1 & 75 &, 365 &, 001 \\
Pair 2 & t2 \& pw2 & 75 &, 407 &, 000 \\
Pair 3 & t3 \& pw3 & 75 &, 070 &, 550 \\
Pair 4 & t4 \& pw4 & 75 &,- 597 &, 000 \\
Pair 5 & t5 \& pw5 & 75 &,- 532 &, 000 \\
Pair 6 & t6 \& pw6 & 75 &,- 092 &, 435 \\
Pair 7 & t7 \& pw7 & 75 &, 143 &, 221 \\
Pair 8 & t8 \& pw8 & 75 &,- 217 &, 062 \\
Pair 9 & t9 \& pw9 & 75 &, 090 &, 444 \\
Pair 10 & t10 \& pw10 & 75 &, 386 &, 001 \\
Pair 11 & t11 \& pw11 & 75 &, 406 &, 000 \\
Pair 12 & t12 \& pw12 & 75 &,- 265 &, 022 \\
Pair13 & t13 \& pw13 & 75 &,- 338 &, 003 \\
Pair 14 & t14 \& pw14 & 75 &,- 679 &, 000 \\
Pair15 & t15 \& pw15 & 75 &,- 028 &, 814 \\
Pair 16 & t16 \& pw16 & 75 &,- 482 &, 000 \\
Pair 17 & t17 \& pw17 & 75 &,- 422 &, 000 \\
Pair18 & t18 \& pw18 & 75 &,- 376 &, 001 \\
Pair 19 & t19 \& pw19 & 75 &, 429 &, 000 \\
\hline
\end{tabular}

Table VI: Correlations between paired samples for the manikin with underwear.

\begin{tabular}{|ll|c|c|c|}
\cline { 3 - 4 } \multicolumn{1}{c|}{} & $N$ & Correlation & Sig. \\
\hline Pair 1 & t1 \& pw1 & 122 &,- 996 &, 000 \\
Pair 2 & t2 \& pw2 & 122 &,- 934 &, 000 \\
Pair 3 & t3 \& pw3 & 122 &,- 539 &, 000 \\
Pair 4 & t4 \& pw4 & 122 &,- 999 &, 000 \\
Pair 5 & t5 \& pw5 & 122 &,- 993 &, 000 \\
Pair 6 & t6 \& pw6 & 122 &,- 993 &, 000 \\
Pair 7 & t7 \& pw7 & 122 &,- 973 &, 000 \\
Pair 8 & t8 \& pw8 & 122 &,- 999 &, 000 \\
Pair 9 & t9 \& pw9 & 122 &,- 990 &, 000 \\
Pair 10 & t10 \& pw10 & 122 &,- 992 &, 000 \\
Pair 11 & t11 \& pw11 & 122 &,- 997 &, 000 \\
Pair 12 & t12 \& pw12 & 122 &,- 619 &, 000 \\
Pair 13 & t13 \& pw13 & 122 &,- 265 &, 003 \\
Pair 14 & t14 \& pw14 & 122 &,- 491 &, 000 \\
Pair 15 & t15 \& pw15 & 122 &,- 277 &, 002 \\
Pair 16 & t16 \& pw16 & 122 &,- 696 &, 000 \\
Pair 17 & t17 \& pw17 & 122 &,- 637 &, 000 \\
Pair 18 & t18 \& pw18 & 122 &,- 639 &, 000 \\
Pair 19 & t19 \& pw19 & 122 &,- 098 &, 285 \\
\hline
\end{tabular}

Similar situation can be observed for the next analysis (Table VII) where firefighter clothing with a flame retardant was tested. Analysis results show high correlation for most pairs, except for pairs 3, 6, 8, 9 and 10. The last analysis (Table VII) shows test results of firefighter clothing with antibacterial protection. Since there is a very high correlation between temperature and heat flow, the null hypothesis can be accepted with a very high probability of $99 \%$. 
Table VII: Correlations between paired samples for firefighter clothing with a flame retardant.

\begin{tabular}{|ll|c|c|c|}
\cline { 2 - 4 } \multicolumn{1}{c|}{} & $\boldsymbol{N}$ & Correlation & Sig. \\
\hline Pair 1 & t1 \& pw1 & 132 &,- 728 &, 000 \\
Pair 2 & t2 \& pw2 & 132 &,- 691 &, 000 \\
Pair 3 & t3 \& pw3 & 132 &,- 156 &, 073 \\
Pair 4 & t4 \& pw4 & 132 &,- 303 &, 000 \\
Pair 5 & t5 \& pw5 & 132 &,- 404 &, 000 \\
Pair 6 & t6 \& pw6 & 132 &,- 116 &, 186 \\
Pair 7 & t7 \& pw7 & 132 &,- 422 &, 000 \\
Pair 8 & t8 \& pw8 & 132 &,- 166 &, 056 \\
Pair9 & t9 \& pw9 & 132 &,- 136 &, 121 \\
Pair 10 & t10 \& pw10 & 132 &,- 135 &, 123 \\
Pair11 & t11 \& pw11 & 132 &,- 288 &, 001 \\
Pair 12 & t12 \& pw12 & 132 &,- 860 &, 000 \\
Pair 13 & t13 \& pw13 & 132 &,- 853 &, 000 \\
Pair 14 & t14 \& pw14 & 132 &,- 675 &, 000 \\
Pair15 & t15 \& pw15 & 132 &,- 817 &, 000 \\
Pair16 132 t16 pw16 & 132 &,- 639 &, 000 \\
Pair 17 & t17 \& pw17 & 132 &,- 469 &, 000 \\
Pair18 & t18 \& pw18 & 132 &,- 503 &, 000 \\
Pair 19 & t19 \& pw19 & 132 &,- 447 &, 000 \\
\hline
\end{tabular}

Table VIII: Correlations between paired samples for firefighter clothing with antibacterial protection.

\begin{tabular}{|c|c|c|c|c|}
\hline & & $N$ & Correlation & Sig. \\
\hline Pair 1 & $\mathrm{t} 1 \& \mathrm{pw} 1$ & 150 &,- 992 & 000 \\
\hline Pair 2 & t2 \& pw2 & 150 &,- 995 & 000 \\
\hline Pair 3 & t3 \& pw3 & 150 &,- 999 & 000 \\
\hline Pair 4 & t4 \& pw4 & 150 &,- 989 & ,000 \\
\hline Pair 5 & t5 \& pw5 & 150 &,- 991 & ,000 \\
\hline Pair 6 & t6 \& pw6 & 150 &,- 991 &, 000 \\
\hline Pair 7 & t7 \& pw7 & 150 &,- 971 &, 000 \\
\hline Pair 9 & t9 \& pw9 & 150 &,- 993 &, 000 \\
\hline Pair 10 & $\mathrm{t} 10 \& \mathrm{pw} 10$ & 150 &,- 990 &, 000 \\
\hline Pair 11 & $\mathrm{t} 11 \& \mathrm{pw} 11$ & 150 &,- 987 & ,000 \\
\hline Pair 12 & $\mathrm{t} 12 \& \mathrm{pw} 12$ & 150 &,- 994 & ,000 \\
\hline Pair 13 & t13 \& pw13 & 150 &,- 997 &, 000 \\
\hline Pair 1 & $\mathrm{t} 14 \& \mathrm{pw} 14$ & 150 &,- 947 &, 000 \\
\hline Pair 15 & t15 \& pw15 & 150 &,- 939 &, 000 \\
\hline Pair 16 & t16 \& pw16 & 150 &,- 994 &, 000 \\
\hline Pair 17 & $\mathrm{t} 17 \& \mathrm{pw} 17$ & 150 &,- 998 & ,000 \\
\hline Pair 18 & $\mathrm{t} 18 \& \mathrm{pw} 18$ & 150 &,- 963 & ,000 \\
\hline Pair 1s & t19 \& pw19 & 150 &,- 967 &, 000 \\
\hline
\end{tabular}

\section{CONCLUSIONS}

The paper presents an attempt to evaluate the measurement accuracy and stability of a measurement system when determining thermal insulation. Thermal insulation is one of the most important parameters that we look for while carrying out tests using a thermal manikin. Since measurement with a thermal manikin is very suitable for determining the thermal insulation of protective and working clothes, we wanted to know how accurate and, consequently, how stable our system with the thermal manikin is.

Measurement accuracy and reliability, which can be, generally speaking, indicated as system stability, were assessed on the basis of statistical analysis. For the analysis of the 
presented problem, descriptive statistics and the Paired sample $t$-test as multivariate analysis of variance were used.

The Paired sample $t$-test gave us a deeper insight into the system stability, which is the aim of our research. Performed analysis and research results show that the system is stable in the most part, but in some measuring points there were some deviations.

With regards to the present results, further research would be useful on weather a greater numerical sample or with more combinations of clothes to avoid demonstrated deviations. Also, minimizing the use of hazardous materials and promoting recycling is important.

\section{REFERENCES}

[1] Mantaras, D. A.; Luque, P. (2015). Assessing motorcyclist protection systems using Finite Element simulations, International Journal of Simulation Modelling, Vol. 14, No. 1, 110-120, doi:10.2507/IJSIMM14(1)10.294

[2] Zavec Pavlinič, D.; Balič, J.; Ficko, M. (2014). Functional products development: Advanced tools and concepts, DAAAM Publishing Series - Manufacturing Technology, DAAAM International, Vienna

[3] Marler, T.; Capdevila, N.; Kersten, J.; Taylor, A.; Wagner, S.; Mackiewicz, J. (2016). Human simulation for task-based survivability analysis, International Journal of Human Factors Modelling and Simulation, Vol. 5, No. 3, 238-262, doi:10.1504/IJHFMS.2016.10000596

[4] Omron. Safety Components, from https://www.ia.omron.com/products/category/safetycomponents/index.html, accessed on 25-01-2016

[5] Harih, G.; Borovinsek, M.; Ren, Z.; Dolsak, B. (2015). Optimal products' hand-handle interface parameter identification, International Journal of Simulation Modelling, Vol. 14, No. 3, 404-415, doi:10.2507/IJSIMM14(3)3.285

[6] Maurice, P.; Padois, V.; Measson, Y.; Bidaud, P. (2016). Experimental assessment of the quality of ergonomic indicators for dynamic systems computed using a digital human model, International Journal of Human Factors Modelling and Simulation, Vol. 5, No. 3, 190-209, doi:10.1504/IJHFMS.2016.10000531

[7] Koren, R.; Palčič, I. (2015). The impact of technical and organisational innovation concepts on product characteristics, Advances in Production Engineering \& Management, Vol. 10, No. 1, 27 39, doi: $10.14743 /$ apem2015.1.190

[8] Ternik, P.; Buchmeister, J. (2015). Buoyancy-induced flow and heat transfer of Power law fluids in a side heated square cavity, International Journal of Simulation Modelling, Vol. 14, No. 2, 238-249, doi:10.2507/IJSIMM14(2)5.293

[9] Abdel-Malek, K.; Yang, J.; Marler, T.; Beck, S.; Mathai, A.; Zhou, X.; Patrick, A.; Arora, J. (2006). Towards a new generation of virtual humans, International Journal of Human Factors Modelling and Simulation, Vol. 1, No. 1, 2-39, doi:10.1504/IJHFMS.2006.011680

[10] Pahole, I.; Valentan, B.; Zavec Pavlinič, D.; Ficko, M.; Balič, J. (2015). Initial study of immersion thermal manikin development and its manufacture from solid blocks, Technical Gazette, Vol. 22, No. 6, 1623-1631, doi:10.17559/TV-20140217093824

[11] Holmér, I. (2004). Thermal manikin history and applications, European Journal of Applied Physiology, Vol. 92, No. 6, 614-618, doi:10.1007/s00421-004-1135-0

[12] Pavlinic, D. Z.; Wissler, E. H.; Mekjavic, I. B. (2011). Using a mathematical model of human temperature regulation to evaluate the impact of protective clothing on wearer thermal balance, Textile Research Journal, Vol. 81, No. 20, 2149-2159, doi:10.1177/0040517511414971

[13] Wu, T.; Tian, R.; Duffy, V. G. (2012). Performing ergonomics analyses through virtual interface design: Validity and reliability assessment, Human Factors and Ergonomics in Manufacturing \& Service Industries, Vol. 22, No. 3, 256-268, doi:10.1002/hfm.20267

[14] Oliveira, A. V. M.; Gaspar, A. R.; Quintela, D. A. (2011). Dynamic clothing insulation. Measurements with a thermal manikin operating under the thermal comfort regulation mode, Applied Ergonomics, Vol. 42, No. 6, 890-899, doi:10.1016/j.apergo.2011.02.005

[15] DuPont. DuPont ${ }^{\mathrm{TM} T h e r m o-M a n}{ }^{\circledR}$, from http://www2.dupont.com/personal-protection/enus/dpt/thermo-man.html/, accessed on 10-06-2012 
[16] Measurement Technology Northwest. Thermetrics, from http://www.mtnw-usa.com/, accessed on 29-10-2012

[17] The Hong Kong Polytechnic University. Institute of Textiles \& Clothing. Walter ${ }^{\text {TM }}$ Sweating Manikin Laboratory, from https://www.polyu.edu.hk/itc/en/about-us/facilities, accessed on 29-10-2012

[18] PT Teknik. Thermal Manikins for Scientific and Industrial Use, from http://pt-teknik.dk/, accessed on 29-10-2012

[19] Qian, X.; Fan, J. (2006). Prediction of clothing thermal insulation and moisture vapour resistance of the clothed body walking in wind, The Annals of Occupational Hygiene, Vol. 50, No. 8, 833842, doi:10.1093/annhyg/mel050

[20] Chen, Y. S.; Fan, J.; Zhang, W. (2003). Clothing thermal insulation during sweating, Textile Research Journal, Vol. 73, No. 2, 152-157, doi:10.1177/004051750307300210

[21] Holmér, I. (1984). Required clothing insulation (IREQ) as an analytical index of cold stress, ASHRAE Transactions, Vol. 90, Part 1, 1116-1128

[22] Zavec, P. D.; Mekjavic, I. B. (2009). Environmental needs co-shape combat clothing systems, Slovenian Armed Forces Magazine, Vol. 17, No. 16, 29-31 (in Slovene)

[23] ISO 15831 (2004). Clothing - physiological effects, Measurement of thermal insulation by means of a thermal manikin, International Organization for Standardization, Geneva, Switzerland

[24] Bogdan, R. C.; Biklen, S. K. (1998). Qualitative Research in Education: An Introduction to Theory and Methods, $3^{\text {rd }}$ ed., Allyn \& Bacon, Needham Heights

[25] Charles, C. M. (1995). Introduction to Educational Research, $2^{\text {nd }}$ ed., Longman, San Diego

[26] Hair, J. F.; Anderson, R. E.; Tatham, R. L.; Black, W. C. (1998). Multivariate Data Analysis, $5^{\text {th }}$ ed., Prentice-Hall International, New Jersey

[27] Nunnally, J. C.; Bernstein, I. H. (1994). Psychometrics Theory, $3^{\text {rd }}$ ed., McGraw-Hill, New York

[28] Kirk, J.; Miller, M. L. (1986). Reliability and Validity in Qualitative Research, Sage Publications, Beverly Hills 